\section{ANÁLISE DOS PROCESSOS DE MODELAÇÃO DE UM MOVIMENTO NO PLANO DESENVOLVIDOS POR ESTUDANTES DO $1^{\circ}$ ANO DO ENSINO MÉDIO}

\section{ANALYSIS OF MODELING PROCESSES OF A MOVEMENT IN THE CARTESIAN PLANE DEVELOPED BY STUDENTS IN THE 1ST YEAR OF HIGH SCHOOL}

\author{
Edson Ferreira da Costa Junior ${ }^{1}$; Karly Barbosa Alvarenga ${ }^{2}$
}

\begin{abstract}
RESUMO
Este trabalho é um recorte de uma pesquisa cujo objetivo é analisar os processos de construção de modelos matemáticos realizados por alunos do primeiro ano do ensino médio, para representar as transformações no plano, especificamente o movimento horizontal. É comum as transformações geométricas, as isometrias, os movimentos rígidos e as simetrias serem apresentados ou com formalidade, ou de maneira simplória. Com o propósito de gerar um trabalho intermediário entre esses dois extremos, elaborou-se uma oficina, com a finalidade de desenvolver o ensino conjunto de álgebra, de geometria e de aritmética para que, assim, os alunos pudessem fazer uso da criatividade e da linguagem matemática e descrever as ações observadas. A oficina foi subsidiada por ideias acerca da modelagem matemática, com a intenção de interpretar e descrever, matematicamente, o movimento de translação horizontal, por meio da manipulação do material concreto, para, desse modo, proporcionar melhor visualização da situação investigada pelos participantes. Inicialmente, realizou-se um teste piloto, com o objetivo de analisar os modelos matemáticos produzidos pelos estudantes do $1^{\circ}$ ano do ensino médio para o movimento de translação, em um contexto de modelagem matemática mediado pelo material concreto. Para a obtenção dos dados, foram empregados ficha de atividade, questionário e gravação em áudio. É importante ressaltar que o objetivo não era encontrar a solução correta, a resposta ideal, mas, sim, possibilitar o contexto de construção do conhecimento fundamentado e significativo em que os estudantes, com o auxílio do professor, eram instruídos a descobrir, criticar e questionar as suas decisões e a criar um modelo com implicações no mundo concreto. Em relação aos resultados iniciais, ao representar o fenômeno, os estudantes demonstraram dificuldades referentes às operações elementares de adição e fizeram uso inadequado da simbologia. Conclui-se que essas dificuldades são reflexos de um ensino mecânico sem produção de significado nas séries anteriores.
\end{abstract}

Palavras-chave: álgebra; aritmética; geometria; ensino; isometrias.

\footnotetext{
${ }^{1}$ Licenciado em Matemática pelo Instituto de Matemática e Estatística da Universidade Federal de Goiás (IME-UFG). Mestrando no Programa de Pós-graduação em Educação em Ciências e Matemática (PPGECM - UFG), Goiânia, Goiás, Brasil. Endereço para correspondência: Rua C-226, Qd 534, Lt 17, Casa 2, Jardim América, Goiânia, Goiás, Brasil. CEP: 74290-080. E-mail: edsonjrpba@ gmail.com.

(iD) ORCID iD: https://orcid.org/0000-0003-2098-4740

${ }^{2}$ Doutora pela Pontifícia Universidade Católica de São Paulo (PUC-SP). Docente na Universidade Federal de Goiás (UFG), Goiânia, Goiás, Brasil. Endereço para correspondência: Rua cel. Ernesto Garcia, Qd 7, Lt 11, apto 602, Vila maria José, Goiânia, Goiás, Brasil. CEP: 74815-460. E-mail: karly@ufg.br.

(iD) ORCID iD: https://orcid.org/0000-0001-7670-8548
} 
Análise dos processos de modelação de um movimento no plano desenvolvidos por estudantes do $1^{\circ}$ ano do ensino médio

\begin{abstract}
This work is an excerpt from a research whose objective is to analyze the processes of construction of mathematical models carried out by students of the first year of high school (14-15 yeas), to represent the transformations of the plane, specifically the horizontal movement. It is common for geometric transformations, isometries, rigid movements and symmetries to be presented either formally or extremely basic. In order to generate an intermediate work between these two extremes, a workshop was created, with the purpose of developing a teaching of algebra, geometry and arithmetic, jointly, so that, thus, students could make use of creativity and language mathematics and describe the observed actions. The workshop was based on ideas about mathematical modeling, with the intention of mathematically interpreting and describing the horizontal translation movement, through the manipulation of concrete material, in order to provide a better view of this movement. Initially, a pilot test was carried out, with the objective of analyzing the mathematical models produced by the students of the 1st year of high school for the translation movement, in a context of mathematical modeling mediated by concrete material. To collect data, an activity sheet, questionnaire and audio recording were used. It is important to emphasize that the objective was not to find the correct solution, the ideal answer, but, rather, to enable the context of construction of grounded and meaningful knowledge in which students, with the help of the teacher. They were instructed to discover, criticize and question their decisions and to create a model with implications that movement situation. In relation to the initial results, when representing the phenomenon, the students demonstrated difficulties regarding elementary addition operations and made inappropriate use of the symbology. It is concluded that these difficulties are reflections of a mechanical teaching without production of meaning in the previous grades.
\end{abstract}

Keywords: algebra; arithmetic; geometry; teaching; isometries. 


\section{Nova possibilidade para o ensino de matemática}

Um dos princípios do ensino de matemática é o prazer da descoberta, mas, há muito tempo, esse aspecto cedeu lugar à memorização. Albuquerque (1954, p. 6), em sua época, asseverou: "nosso ensino apela muito para a memória, e, como memória não significa inteligência, a criança de inteligência normal ou superior deixará de aprender coisas elementares se não possui boa memória". Diante dessa realidade, autores, como Silva, Sousa e Medeiros (2020) ressaltam a importância de ressignificar o ensino. Vejamos os seus argumentos, resultados de pesquisa bibliográfica:

Os resultados deste arcabouço teórico apontam para a necessidade de ressignificação do processo de ensino e aprendizagem, o que requer investimentos, capacitações e formações para que os profissionais, muitos ainda destes atrelados às técnicas básicas de ensino, possam oxigenar novas possibilidades de ensinar, com práticas de qualidade que objetivem o desenvolvimento real e satisfatório dos estudantes para além do contexto escolar. (SILVA; SOUSA; MEDEIROS, 2020, p. 2)

A perspectiva desses autores reforça a importância da nossa proposta, que contém objetivos de ressignificar o ensino para além da memorização, contextualizar o conteúdo e aproximá-lo do mundo concreto do estudante, inclusive fora do ambiente escolar. Cometeríamos uma falha se fizéssemos algo diferente disso.

Dessa maneira, faz-se necessário, em relação às práticas cotidianas de nossos alunos, descobrir formas mais simples e dinâmicas para o ensino, independentemente da área de conhecimento. Afinal, "em geral, falhas em leitura e em noções básicas de Matemática originam o insucesso do estudante”. (ALBUQUERQUE, 1954, p. 6).

Nesse sentido, alguns autores ressaltam a importância de um trabalho dinâmico que extrapole o tradicional, com o intuito de romper com a mecanização do ensino, em específico o de Geometria. Ribeiro e Jahn (2015, p. 40), de acordo com esse ponto de vista, afirmam:

Ocorre ainda que, de um modo geral, as abordagens para o ensino e a aprendizagem de Geometria na Educação Básica são feitas de maneira estática. As figuras são apresentadas sem que se dê movimento a elas, o que pode dificultar a compreensão de suas características ou propriedades por parte dos alunos.

Propomos, assim, com a modelagem matemática, auxiliada pela álgebra, pela geometria e pela aritmética, contribuir para a superação de dificuldades no processo de ensino das isometrias, que estão presentes no cotidiano. Essas formas podem ser 
observadas, por exemplo, na construção dos padrões artísticos, como o tratamento dos polígonos regulares, nas figuras geradas por livros de espelhos, ou seja, por reflexões, na classificação dos quadriláteros quanto às suas simetrias, na construção dos polígonos regulares inscritos numa circunferência ou em frisos e padrões por iteração de um conjunto de isometrias geradoras dessas figuras (BASTOS, 2006). Na física, notadamente, observam-se as transformações que mantêm o volume. Na matemática, elas têm o significado pautado nas transformações que preservam distâncias entre pontos, ângulos, ou seja, são responsáveis por transformar uma figura, ou forma, em outra geometricamente igual.

Quando analisamos a Base Nacional Comum Curricular (BNCC), especificamente a sexta competência, que ressalta as habilidades necessárias para o ensino fundamental relativas à área da matemática, observamos a necessidade do desenvolvimento de atividades referentes a múltiplos contextos em relação ao cotidiano do estudante, reais ou imaginários (BRASIL, 2017). Em vista disso, a modelagem matemática pode contribuir para a inserção do contexto em sala de aula, além de ser um meio para dar significado ao ensino e associá-lo ao mundo real. Ademais, durante esse processo, a construção do conhecimento é o fator principal, em que o estudante, com a ajuda do professor, será instruído a descobrir, criticar, questionar as próprias conclusões e, assim, construir o seu conhecimento fundamentado e significativo.

Consequentemente, com o uso de materiais manipuláveis e aplicáveis a situações reais, podemos desenvolver o senso crítico do estudante e auxiliá-lo na capacidade de constatar o significado da matemática no cotidiano. Na nossa proposta, o resultado final do trabalho com a modelagem matemática é a construção de um modelo que represente o movimento de translação na horizontal.

Este trabalho partiu da seguinte questão investigativa: quais são os modelos matemáticos para o movimento de translação apresentados pelos estudantes do $1^{\circ}$ ano do ensino médio de uma escola pública de Goiânia-Go, após participarem de uma oficina com material concreto? O objetivo principal desta pesquisa é analisar os processos de construção de modelos matemáticos produzidos pelos estudantes do $1^{\circ}$ ano do ensino médio para o movimento de translação, em um contexto de modelagem matemática mediado por material manipulável. O nosso intuito é propor caminhos que levem o 
Análise dos processos de modelação de um movimento no plano desenvolvidos por estudantes do $1^{\circ}$ ano do ensino médio

estudante a ter o prazer da descoberta das próprias conclusões, a desenvolver sua capacidade de aprendizagem, a elaborar modelos e a obter conhecimento matemático.

\section{O que consideram algumas pesquisas acerca da temática}

Neste tópico, realizamos breve apresentação de outras pesquisas sobre a temática. De acordo com a nossa proposta, analisamos os modelos matemáticos desenvolvidos pelos estudantes para o movimento de translação. Os autores discutem as possibilidades de um trabalho com a modelagem matemática e afirmam ser ela fundamental para a obtenção de novos conhecimentos e habilidades.

Biembengut e Hein (2004) defendem dois tipos de abordagem no ensino possíveis em qualquer escolaridade. No primeiro, o professor pode eleger um tema de alguma área do conhecimento de interesse dos alunos e elaborar um modelo matemático adaptado ao ensino. No segundo, o intuito é orientá-los para um trabalho de modelação, de maneira a criar condições para que sejam capazes de investigar e elaborar modelos matemáticos aplicados a alguma área (BIEMBENGUT; HEIN, 2004). Ambas as vertentes têm como premissa a promoção de conhecimentos e habilidades matemáticos para aplicá-los também em outros contextos e, assim, fornecer elementos para o aluno desenvolver o seu potencial e promover o seu pensamento crítico e independente. Pelo exposto, identificase que a nossa proposta tem similaridade com a primeira abordagem.

Para os autores Biembengut e Hein (2004) e Bassanezi (2009), o resultado do trabalho com a modelagem é o modelo matemático. Os autores apresentam o seu conceito vinculado ao processo de resolução, com símbolos e relações matemáticas a representar o fenômeno analisado.

A modelagem é uma ferramenta de estudo de situações e fenômenos presentes no mundo real, com propósito de obter resultados e conclusões. Em nossa pesquisa, é utilizada para a análise de um fenômeno que não é considerado propriamente do cotidiano, mas que está presente e passa despercebido aos olhos de muitos. A perspectiva que expõe Machado (2009) sobre a relação entre o abstrato e o concreto justifica a nossa escolha.

A possibilidade de as abstrações matemáticas serem tratadas como algo em si, desvinculadas do substrato empírico que as engendrou, não pode ser negada. Afinal, assim se processam profícuos trabalhos de numerosos matemáticos que, assumindo uma divisão do trabalho que embasa toda a sociedade de que 
participam, deixam para outros a tarefa de discutir, a posteriori, os possíveis vínculos do que produzem com o mundo que está por aí, de procurar aplicações para o que, ingenuamente, acreditam produzir de forma livre e desinteressada. Trata-se, muitas vezes, de uma opção que deve ser respeitada tal como a avestruz, que esconde a cabeça no solo; é o seu modo de interagir com o mundo real (MACHADO, 2009, p. 53).

Dessa forma, não buscamos a alternativa correta ou a resposta ideal para as questões abordadas na oficina, mas, sim, o raciocínio crítico sobre o conjunto da eventual problemática subjacente a tais questões, com uso de ferramentas matemáticas para a compreensão da realidade e a elaboração de um modelo com resultados aplicáveis na realidade concreta. Para a construção de um modelo matemático, são necessárias algumas ferramentas, tal como explicam Bassanezi (2009) e Biembengut e Hein (2004). Para a construção do nosso, empregamos a álgebra, a aritmética e a geometria. Entretanto, como evidenciamos a seguir, muitos alunos apresentaram dificuldades.

Castro (2012) ratifica nossa observação. Em um levantamento realizado pelo referido autor, constatou-se que o conhecimento algébrico obtido por parte dos alunos era insatisfatório. A justificativa apresentada relaciona-se aos obstáculos e às dificuldades inerentes ao objeto que se devem, em grande parte, à natureza da álgebra, a sua linguagem ou a outros elementos que a compõem. É preciso ressaltar, contudo, que a qualidade do ensino está vinculada aos traços do currículo e dos professores que a colocam em prática (CASTRO, 2012).

Lins e Gimenez (2000, p. 12) asseveram que "a aritmética e a álgebra constituem, junto com a geometria, a base da matemática escolar". Entretanto, no currículo, os conceitos aritméticos correspondem a relações quantitativas, e esquece-se, frequentemente, de que o uso da aritmética inclui as representações, as significações, o emprego racional e adequado de regras, a análise do porquê dos algoritmos e dos elementos conceituais e a elaboração de conjecturas (LINS; GIMENEZ, 2000).

Para Ponte (2006), no centro da aritmética, estão os números e suas operações; na geometria, estão os objetos geométricos, as abstrações do plano e do espaço e suas transformações; na álgebra, as relações matemáticas abstratas, que podem ser equações, inequações, funções ou estruturas demarcadas por operações ou relações em conjuntos. Essas ferramentas foram utilizadas para o desenvolvimento do modelo matemático de nossa pesquisa. 
Quando praticamos o ensino com essas três áreas concomitantemente, fortalecemos a aprendizagem delas e facilitamos a compreensão ampla dos significados conceituais (OLIVEIRA; LAUDARES, 2015). Além disso, “o uso da Geometria, para contextualizar o ensino da Álgebra no ensino fundamental, pode tornar seu ensino mais interessante e motivador" (OLIVEIRA; LAUDARES, 2015, p. 7).

Para representar o movimento de translação, é necessário, portanto, o estudante fazer uso da álgebra, da aritmética e da geometria. Nessa mesma linha, seria útil empregar a linguagem concisa, da simbologia, das operações e das relações que representem a situação analisada mediante um modelo. Com isso, transpõe-se o problema estudado à realidade da matemática, o qual deve ser tratado com teorias e técnicas da própria ciência.

$\mathrm{O}$ uso do material concreto tem como finalidade facilitar a análise do fenômeno estudado pela modelagem. Ademais, isso possibilita o contato direto do aluno como sujeito responsável pela própria aprendizagem, tal como exposto pelos autores Rêgo M. e Rêgo G. (2006):

O material concreto exerce um papel importante na aprendizagem. Facilita a observação e análise, desenvolve o raciocínio lógico, crítico e científico, é fundamental para o ensino experimental e é excelente para auxiliar o aluno na construção de seus conhecimentos (RÊGO M.; RÊGO G., 2006, p. 61).

Nesse sentido, o material concreto serve de suporte para a construção do modelo em atribuição à modelagem matemática e auxilia o desenvolvimento do raciocínio crítico e científico do estudante, a observação e a experimentação do fenômeno em análise. Assim, com a modelagem matemática, é possível superar as dificuldades no processo de ensino e fazer uso da simbologia, das relações e das operações, para descrever o fenômeno estudado mediante a álgebra, a aritmética e a geometria. Além disso, é necessário complementar essa metodologia com a utilização dos materiais manipuláveis, para auxiliar a aprendizagem dos estudantes e facilitar a visualização da situação analisada.

\section{A abordagem utilizada para coleta e análise dos dados}

A proposta desenvolvida tem traços da pesquisa etnográfica discutida por André (2013), na qual são aliados elementos inerentes às abordagens qualitativas com respaldo numérico. Assim, realizamos um trabalho de campo diretamente em contato com a situação e o ambiente da pesquisa. Segundo a autora, a pesquisa do tipo etnográfico exige 
um contato direto do pesquisador com a situação analisada, afetando-a e sendo afetado por ela, pois esse contato permite reconstruir os processos e as relações que configuram a experiência escolar (ANDRÉ, 2013).

Na nossa pesquisa, realizamos um teste piloto com 14 alunos do $1^{\circ}$ ano do ensino médio, em uma escola pública de Goiânia, com o intuito de avaliar os dados iniciais coletados e potencializar a atividade, que ocorreu em três dias consecutivos, com três aulas no primeiro, três no segundo e duas no terceiro dia. A atividade consistiu na construção de três modelos que representavam a situação analisada, o movimento de translação. As duas situações iniciais, o movimento de translação vertical e diagonal, foram apresentadas pelo professor pesquisador e discutidas com os estudantes, assim como os seus modelos representativos.

Durante a oficina, exemplificamos os movimentos na direção vertical e diagonal com apoio do material concreto e, por fim, apresentamos o modelo matemático que representava cada um. A atividade consistia em realizar movimentos de forma livre, com apoio de uma figura sobre folha A4 fixa na mesa e, assim, construir o modelo que representava aquele movimento. Por fim, a terceira situação, realizada em casa, em dupla, foi referente à construção de um modelo que representasse o movimento horizontal.

De acordo com a nossa proposta, nas duas situações iniciais para o movimento de translação vertical e diagonal, o professor pesquisador foi o responsável pela elaboração do problema. As etapas seguintes foram desenvolvidas em conjunto com os estudantes, exceto a terceira situação, na qual o professor apresentou informações necessárias para a resolução, entretanto sua execução foi responsabilidade dos alunos.

De acordo com o enfoque utilizado para os movimentos na direção vertical e diagonal, a terceira situação era similar e consistia na descrição do movimento horizontal com palavras e uso da simbologia, das relações e das operações matemáticas, com direção horizontal e sentido à direita ou à esquerda, a critério de cada dupla. Assim, os estudantes deveriam citar três exemplos:

- O primeiro seria relacionado à análise em relação ao ponto central da figura, com constante fixa de quatro unidades de deslocamento;

- O segundo seria referente a um ponto qualquer da figura $(a, b)$ com constante fixa de quatro unidades de deslocamento;

- O terceiro seria um ponto qualquer da figura $(a, b)$ para uma constante de deslocamento qualquer $(0, k)$. 
Edson Ferreira da Costa Junior e Karly Barbosa Alvarenga

Análise dos processos de modelação de um movimento no plano desenvolvidos por estudantes do $1^{\circ}$ ano do ensino médio

Além disso, os estudantes deveriam realizar um relato de todo o processo. Com base nos dados da gravação em áudio da oficina, utilizados como forma de registro de ocorrências durante o desenvolvimento da pesquisa, foi possível documentar alguns problemas relacionados à dinâmica da entrega do material, à ausência dos estudantes e à necessidade do redirecionamento do conteúdo, conforme indica o quadro 1 .

Quadro 1: Problemas iniciais na coleta de dados

\begin{tabular}{|c|l|}
\hline Problemas iniciais & Descrição e solução \\
\hline $\begin{array}{c}\text { Dinâmica da entrega do } \\
\text { material }\end{array}$ & $\begin{array}{l}\text { Em razão da quantidade, a entrega dos materiais demandou muito tempo } \\
\text { durante a oficina. Logo, uma possibilidade para evitar esse tipo de } \\
\text { situação seria qualificar um monitor, para auxiliar os processos. }\end{array}$ \\
\hline $\begin{array}{c}\text { Ausência dos estudantes } \\
\text { Necessidade do } \\
\text { redirecionamento do } \\
\text { conteúdo }\end{array}$ & $\begin{array}{l}\text { Por causa da duração da oficina, alguns alunos frequentaram-na apenas } \\
\text { parcialmente. Para solucionar esse problema, sintetizamos o conteúdo } \\
\text { trabalhado, de forma a reduzir o tempo de oficina para um dia. }\end{array}$ \\
\hline $\begin{array}{l}\text { No teste piloto, desenvolvemos os movimentos na direção vertical e } \\
\text { diagonal. Avaliamos que o movimento diagonal necessitava de muitas } \\
\text { ferramentas para a sua resolução e demandaria muito tempo para a sua } \\
\text { compreensão. Assim, esse conteúdo foi retirado da oficina. }\end{array}$ \\
\hline
\end{tabular}

Fonte: dados da pesquisa.

Em seguida, realizamos uma readaptação do material da oficina para coleta posterior e complementação dos dados da pesquisa, que está em fase de finalização. Outro material analisado foi a ficha de atividade desenvolvida pelos estudantes, em que examinamos o tipo de escrita utilizada no modelo, o relato sobre o processo, a simbologia, as operações envolvidas e as exemplificações. Esses dados foram categorizados de acordo com a análise de conteúdo de Bardin (2006).

As categorias encontradas na atividade desenvolvida pelos alunos foram: enfoque geométrico, enfoque aritmético e enfoque algébrico. Diante disso, classificamo-los de acordo com as características em comum. Dessa forma, construímos o quadro 2 com a descrição de cada um e sua relação com o modelo matemático.

Quadro 2: Descrição de cada enfoque e sua relação com o modelo matemático

\begin{tabular}{|c|c|c|}
\hline & Descrição & Relação com o modelo matemático \\
\hline Enfoque geométrico & $\begin{array}{l}\text { O plano, suas transformações e } \\
\text { a reta direção para demonstrar o } \\
\text { movimento na horizontal. }\end{array}$ & $\begin{array}{l}\text { Usar o plano cartesiano na demonstração do } \\
\text { movimento. } \\
\text { Identificar a reta direção do movimento. }\end{array}$ \\
\hline Enfoque aritmético & $\begin{array}{l}\text { Operações numéricas e relações } \\
\text { quantitativas para descrever o } \\
\text { movimento na horizontal. }\end{array}$ & $\begin{array}{l}\text { Empregar operações numéricas ou abstratas } \\
\text { na representação do movimento, ou seja, a } \\
\text { adição ou a subtração das coordenadas em } \\
\text { relação à unidade de deslocamento. }\end{array}$ \\
\hline Enfoque algébrico & $\begin{array}{l}\text { Uso de relações } \\
\text { abstratas } \quad \text { ou }\end{array}$ & $\begin{array}{l}\text { Usar operações abstratas na representação } \\
\text { do movimento no plano. Outro aspecto é a }\end{array}$ \\
\hline
\end{tabular}


Edson Ferreira da Costa Junior e Karly Barbosa Alvarenga

Análise dos processos de modelação de um movimento no plano desenvolvidos por estudantes do $1^{\circ}$ ano do ensino médio

demarcadas por operações entre adição ou a subtração das coordenadas em essas relações. relação à unidade de deslocamento.

Fonte: dados da pesquisa.

Alguns dados do teste piloto foram descartados, por apresentar elementos idênticos, o que inviabilizou a identificação do autor original. Essa relação está apresentada no quadro 3.

Quadro 3: Quantitativo dos dados do teste piloto

\begin{tabular}{|l|l|}
\hline Dados & Quantitativo \\
\hline Número de participantes & 14 participantes \\
\hline Tempo de oficina & 6 h 30 min \\
\hline Trabalhos avaliados & 5 trabalhos de 5 duplas \\
\hline Trabalhos descartados & 2 trabalhos de 2 duplas \\
\hline Questionários avaliados & 10 questionários individuais \\
\hline Questionários descartados & 4 questionários \\
\hline
\end{tabular}

Fonte: dados da pesquisa.

Houve um número maior de participantes no teste piloto, entretanto, em razão de algumas circunstâncias, os dados foram descartados em três diferentes situações: nos casos em que os participantes faltaram, pelo menos, uma vez à oficina e não participaram de todo o processo; nos casos em que os questionários, a ser respondidos de forma individual, apresentaram respostas idênticas; nos casos em que alguns trabalhos apresentaram elementos semelhantes na construção do modelo, por isso foram excluídos da análise da pesquisa. Dessa forma, houve um número menor de avaliados em relação ao quantitativo coletado. O mesmo ocorreu com os questionários.

\section{Discussões e resultados do teste piloto}

Com base na análise da atividade desenvolvida pelos estudantes, obtivemos os enfoques geométrico, aritmético e algébrico na construção do modelo matemático. Contudo, entre os dados coletados, apresentaremos apenas uma atividade (um modelo matemático) para efeito de discussão dos resultados do trabalho.

As atividades desenvolvidas pelos estudantes NV, S, conforme indica a figura 1, apresentam os três enfoques na construção do modelo matemático. A atividade consistia na análise do movimento na direção horizontal, mas, de acordo com o enfoque aritmético e algébrico, identificamos um movimento na direção vertical, como será evidenciado a seguir. 
Figura 1: Enfoque aritmético e algébrico produzido pelos estudantes NV, S

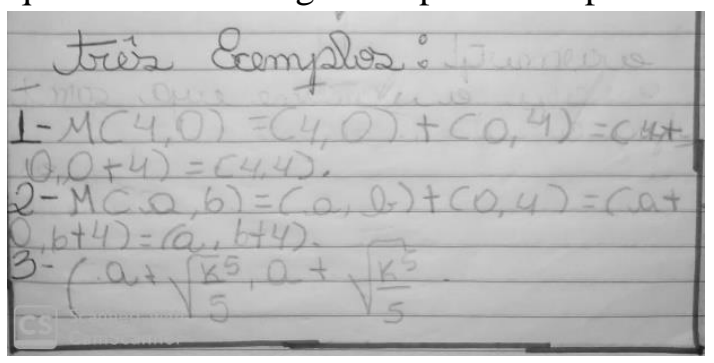

Fonte: dados da pesquisa.

Os estudantes utilizaram um exemplo discutido durante a oficina para o movimento na direção vertical, que se encontra no material de apoio. Exceto o terceiro item da atividade, no qual a generalização do ponto tem proximidade com a do movimento diagonal, eles diferenciaram um modelo vertical de um horizontal e não conseguiram compreender os elementos que permeiam esses dois.

Acreditamos que essas dificuldades são proporcionadas pelo foco do ensino nas séries anteriores que enfatiza a memorização e a mecanização de fórmulas, o que pode gerar dificuldades posteriores na resolução de problemas. Desse modo, os estudantes não conseguem fazer uso das ferramentas que aprenderam, tal como expõem Oliveira e Laudares (2015, p. 2):

Esse tipo de abordagem reflete diretamente na compreensão das operações elementares e na aprendizagem significativa da Álgebra, acarretando dificuldades associadas à resolução de problemas dentro de um contexto do cotidiano e em outros níveis de ensino.

Novamente, pela difusão do ensino mecânico, os estudantes fazem uso de ferramentas, sem ter o mínimo conhecimento sobre elas e sobre qual será a sua utilidade na resolução de problemas. Oliveira e Laudares $(2015$, p. 2) explicam que:

\footnotetext{
O ensino da matemática de forma tradicional, como uma simples transmissão de conhecimentos através de técnicas desprovidas de significados e a repetição do algoritmo sem sentido algum, dão lugar à memorização, repetição sem a garantia do sucesso da aprendizagem.
}

Observamos esse insucesso diante do fato de que o enfoque geométrico não foi utilizado para a construção do modelo para o movimento horizontal, item avaliado na atividade. $\mathrm{O}$ esboço do plano cartesiano apresentado ficou incorreto pela posição numérica. Outro fato observado está relacionado à não utilização do enfoque geométrico 
como forma de registro; não foram destacadas, portanto, a posição inicial ou a final do ponto analisado, mesmo após a aplicação da unidade de deslocamento. Realizou-se, tão somente e incorretamente, o esboço do plano, sem dar nenhuma utilidade a ele.

Esse tipo de aprendizagem mecânica e sem significado foi reforçada mais uma vez quando os estudantes fizeram o detalhamento da atividade de forma sintética. Nesse contexto, eles explicam: "Começamos a fazer o gráfico com o plano cartesiano; em seguida, escrevemos os números no gráfico de forma correta; depois, fizemos o movimento com a figura; por último, descobrimos o valor exato do movimento" (Figura 2). Tal como observado anteriormente, o plano cartesiano está incorreto, não há o gráfico (enfoque geométrico) na situação analisada e, muito menos, o valor exato do movimento.

Figura 2: Descrição do movimento produzido pelos estudantes NV, S

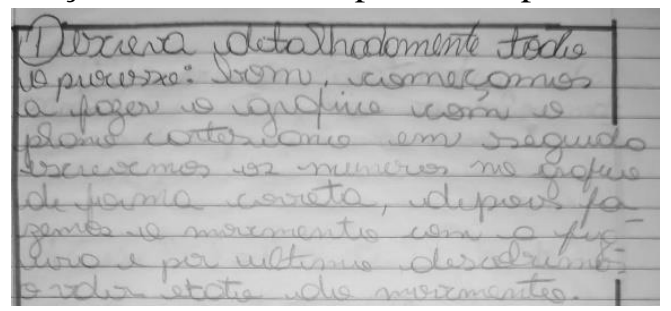

Fonte: dados da pesquisa.

Com base nesse resultado, supomos que os estudantes podem não ter compreendido a atividade ou não ter conhecimento suficiente quanto ao uso dos enfoques geométrico, aritmético e algébrico, para entender que o movimento vertical é diferente do horizontal. Dado que as variáveis envolvidas no processo são diferentes para o movimento retilíneo vertical, temos alterações na ordenada da unidade de deslocamento, com abscissa fixa. No movimento horizontal, ocorre o contrário disso.

Os estudantes NV e S apresentaram dados semelhantes no questionário. Ambos declararam não gostar de matemática e nunca ter descrito algum fenômeno matematicamente; além disso, associam a linguagem ao uso de números e letras, e a matemática em si, a cálculos numéricos. Esses elementos advêm da perspectiva de aprendizagem mecânica e sem significado, tal como asseveram Oliveira e Laudares (2015). Apesar dessa visão equivocada, o fato é que, com a matemática, é possível analisar e estudar situações. Um exemplo disso é a atividade desenvolvida, que permite os fenômenos serem descritos matematicamente (BASSANEZI, 2009; BIEMBENGUT; HEIN, 2004). 
Apesar de a geometria, a aritmética e a álgebra serem consideradas basilares da matemática escolar (LINS; GIMENEZ, 2000), constatamos, pela atividade realizada, que grande parte dos alunos tem dificuldades no seu uso, principalmente, para descrever uma situação problema e relacioná-la a essas três áreas. Observamos que as dificuldades perpassam os três enfoques desde aspectos simples, como a ordenação dos números, até outros de maior complexidade, como a escolha da unidade de deslocamento horizontal, relacionada ao enfoque geométrico.

Além disso, destacamos que um dos motivos para o insucesso na aprendizagem da matemática é o trabalho disjunto entre as três áreas, álgebra, aritmética e geometria, e um fator importante no processo de superação dessa tendência é o trabalho do professor. Oliveira e Laudares (2015, p. 4) reforçam a importância do trabalho conjunto. Em relação a esse assunto, eles assim se posicionam:

\footnotetext{
Com o objetivo de que os estudantes alcancem uma formação de conceitos algébricos satisfatórios e para que obtenham um desenvolvimento do pensamento algébrico consistente, o ensino da Álgebra deve não só estar articulado com os conceitos aritméticos desde os anos iniciais do Ensino Fundamental, mas enfatizar as várias concepções da álgebra também dentro da Geometria, visando assim, um efetivo desenvolvimento do pensamento abstrato e a capacidade para generalizar os conceitos nas séries posteriores à educação básica.
}

Nesse caso, a atividade desenvolvida é de grande importância para a construção do conhecimento, pois, além de estimular os estudantes a discutir e a investigar a situação, incentivou-os a utilizar as ferramentas matemáticas e relacionar a álgebra, a aritmética e a geometria na construção do modelo.

Como já apontado, os estudantes não conseguiram fazer uso dos três enfoques para representar o fenômeno analisado na nossa pesquisa. Apesar de os autores Lins e Gimenez (2000) afirmarem a importância dessas três áreas para a base da matemática escolar, verificamos dificuldades na apropriação dos conceitos trabalhados na atividade, mesmo após esses estudantes chegarem à última etapa da educação básica.

Foi possível verificar, em alguns casos, que os estudantes apresentaram exemplos de um movimento vertical diferente da proposta da atividade. Uma hipótese para a ocorrência de tal fato pode estar relacionada à forma como foi utilizado o material entregue a cada estudante, que era apenas para consulta e não deveria ser reproduzido. Afinal, na construção do modelo matemático para o movimento horizontal, existia 
diferença para a escolha da unidade de deslocamento. Porém, muitos utilizaram a mesma unidade tanto na direção vertical quanto na horizontal.

Constatamos, pois, um déficit de aprendizagem em relação às operações básicas de adição, pois, mesmo com a resolução de exemplos, os alunos realizaram as operações de forma incorreta. Também, há o uso inadequado da simbologia para as operações, como o sinal de igualdade e adição. Isso corrobora o estudo de Castro (2012) acerca da insuficiência quanto aos conhecimentos algébricos. Ademais, essas dificuldades são um reflexo das abordagens mecânicas no ensino (OLIVEIRA; LAUDARES, 2015; ALVARENGA; BRASIL; GUADAGNINI, 2020; ALVARENGA; BRASIL, 2020). Nesse sentido, reiteramos a necessidade de propor situações contextualizadas para o estudo das operações aritméticas básicas. Além disso, destacamos a necessidade de mais pesquisas com abordagens na construção de modelos relacionados às isometrias, sobre trabalhos que desenvolvam, de forma conjunta, a aritmética, a álgebra e a geometria. Para a construção do material para essa oficina, foi realizada uma investigação árdua para chegar-se ao modelo representativo do movimento de translação.

Ressaltamos, inclusive, a importância de um trabalho na perspectiva da modelagem matemática para que os nossos estudantes transformem elementos da realidade em problemas matemáticos e consigam resolvê-los com auxílio de ferramentas da área, a fim de que se envolvam, efetivamente, no processo de construção do conhecimento, pois, somente dessa maneira, eles estarão motivados a descobrir, criticar e questionar os resultados encontrados. Além disso, esse tipo de trabalho abre portas para metodologias de ensino alternativas que possibilitam extrapolar o uso comum do quadro e do giz e analisar, cientificamente, determinada situação, tornando possível, assim, substituir a visão ingênua de dada realidade por um posicionamento crítico e abrangente que facilite e racionalize o pensamento e que possibilite, enfim, agir e transformar o contexto (BASSANEZI, 2009).

\section{Referências}

ALBUQUERQUE, Irene de. Metodologia da matemática. 2 ed. Rio de Janeiro: Conquista, 1954.

ALVARENGA, Karly; BRASIL, Débora Barbosa. Moura. Análise de algumas compreensões algébricas de estudantes da Educação Superior. In: NEVES, Regina da 
Análise dos processos de modelação de um movimento no plano desenvolvidos por estudantes do $1^{\circ}$ ano do ensino médio

Silva Pina; DÖRR, Raquel Carneiro. Ensino de matemática. Jundiaí: Paco Editorial, 2020.

ALVARENGA, Karly; BRASIL, Débora Barbosa. Moura; GUADAGNINI, Míriam Rocio. Erros algébricos insistentes: uma análise de 1910 e outra de 2019. In: BRAGA, Dalvirene; NOLETO, Carine; NOGUEIRA, Cleia. Investigações em ensino de matemática. Jundiaí: Paco Editorial. 2020

ANDRÉ, Marli. Etnografia da prática escolar. São Paulo: Papirus, 2013.

BARDIN, Laurence. Análise de conteúdo. Trad. Luís Antero Reto e Augusto Pinheiro. São Paulo: Edições 70, 2006.

BASSANEZI, Rodney Carlos. Modelagem matemática: um método científico ou uma estratégia de ensino aprendizagem. São Paulo: Contexto. 2009.

BASTOS, Rosa. Notas sobre o ensino da geometria. In: Quadrante. maio/junho, 2006.

BIEMBENGUT, Maria Salett; HEIN, Nelson. Modelación matemática y los desafíos para enseñar matemática. In: Educación matemática, v. 16, n. 2, p. 105-125, ago. 2004.

BRASIL, Mistério da Educação. Base nacional comum curricular. Brasília: MEC, Secretaria de Educação Básica, 2017.

CASTRO, Encarnación. Dificultades en el aprendizaje del álgebra escolar. In: Investigación en Educación Matemática, 16, 2012, Granada, Anais. Jaén: SEIEM, 2012. p. 75-94.

LINS, Rômulo Campos; GIMENEZ, Joaquim. Perspectivas em aritmética e álgebra para o século XXI. São Paulo: Papirus, 2000.

MACHADO, Nílson José. Matemática e realidade. 7. ed. São Paulo: Cortez, 2009.

OLIVEIRA, Silvânia Cordeiro; LAUDARES, João Bosco. Pensamento algébrico: uma relação entre álgebra, aritmética e geometria. In: Encontro Mineira de Educação Matemática, 7. Anais EMEM, São João Del Rei, 2015, p. 1-10.

PONTE, João Pedro. Números e álgebra no currículo escolar. In: VALE, Isabel; PIMENTEL, Teresa; BARBOSA, Ana et al. (org.) Números e álgebra na aprendizagem da matemática e na formação de professores. Lisboa: SEM-SPCE, 2006. p. 5-27.

RÊGO, Rômulo Marinho; RÊGO, Rogéria Gaudêncio. Desenvolvimento e uso de materiais didáticos no ensino de matemática. In: LORENZATO, Sérgio (org.). 0 laboratório de ensino de matemática na formação de professores. Campinas: Autores Associados, 2006. p. 39-56. 
Análise dos processos de modelação de um movimento no plano desenvolvidos por estudantes do $1^{\circ}$ ano do ensino médio

RIBEIRO, Marco Antônio da Silva; JAHN, Ana Paula. Transformações geométricas planas: um estudo experimental e dinâmico. Anais. São Paulo: IME-USP, 2015.

SILVA, Ana Gisnayane Sousa; SOUSA, Francisco Jucivânio Félix de; MEDEIROS, Jarles Lopes de. O ensino da matemática: aspectos históricos. In: Research, Society and Development, v. 9, n. 8, 2020.

Recebido em: 26 / 02 / 2021

Aprovado em: 17 / 04 / 2021 